\title{
Role of Sn impurity on electronic topological transitions in 122 Fe-based superconductors
}

\author{
Haranath Ghosh ${ }^{\mathrm{a}, \mathrm{b}, 1}$, Smritijit Sen ${ }^{\mathrm{a}}$ \\ ${ }^{a}$ Homi Bhabha National Institute, Anushaktinagar, Mumbai 400 094, India. \\ ${ }^{b}$ Indus Synchrotrons Utilization Division, Raja Ramanna Centre for Advanced \\ Technology, Indore -452013, India.
}

\begin{abstract}
We show that only a few percentage of Sn doping at the Ba site on $\mathrm{BaFe}_{2} \mathrm{As}_{2}$, can cause electronic topological transition, namely, the Lifshitz transition. A hole like $\mathrm{d}_{x y}$ band of Fe undergoes electron like transition due to $4 \%$ Sn doping. Lifshitz transition is found in $\mathrm{BaFe}_{2} \mathrm{As}_{2}$ system around all the high symmetry points. Our detailed first principles simulation predicts absence of any Lifshitz transition in other 122 family compounds like $\mathrm{SrFe}_{2} \mathrm{As}_{2}, \mathrm{CaFe}_{2} \mathrm{As}_{2}$. This work bears practical significance due to the facts that a few percentage of Sn impurity is in-built in tin-flux grown single crystals method of synthesizing 122 materials and inter-relationship among the Lifshitz transition, magnetism and superconductivity.
\end{abstract}

Keywords: Fe-based superconductors, Lifshitz transition, Fermi Surface

\section{Introduction}

The phase diagrams of Fe-based superconductors (SCs) comprise of a large number of exotic phases like spin density wave (SDW) [1, 2, 3], orbital order [4, 5, 6, 7], structural transition [8, 9], nematic order [10, 11, 12] etc. These phases are sensitive to temperature, pressure, doping concentration as well as internal crystallographic structures of the materials [13, 14, 6, 15, 16]. In order to perceive insight on the origin of these intriguing features, enormous efforts have already been made by researchers through their experimental and theoretical investigations on electronic structures of these materials

\footnotetext{
${ }^{1}$ Corresponding author : hng@rrcat.gov.in
} 
[17, 18, 19, 20, 21, 22, 23, 24]. Among Fe based SCs, 122 family attribute to a large number of anomalies in structural, magnetic and superconducting properties including electronic topological transition (ETT) or Lifshitz transition [25, 1, 26, 27, 28]. Unlike oxypnictides, high quality large single crystals of 122 Fe-based materials can be easily grown by the flux method. One of the popular methods for synthesizing 122 single crystals is tin-flux method. Single crystals grown by this tin-flux method, contain unwanted Sn impurity. A sufficient number of literatures is available raising the issue of $\mathrm{Sn}$ contamination in 122 single crystals. Those investigations broach the point of irrefutable alteration in the physical properties of 122 systems in presence of Sn impurity by several experiments [29, 30, 31]. Absence of any fruitful and conclusive theoretical study regarding the effect of Sn contamination in the 122 systems, make things harder to understand the observed anomalies in various physical properties. On the other hand, theoretical study of Lifshitz transition is of fundamental importance considering its influence in many emergent branches of condensed matter physics e.g., in topological Dirac semi metal [32], bilayer graphene [33], quantum Hall liquids [34], high $\mathrm{T}_{c}$ cuprates [35] etc. (Note, the later systems are known to be strongly correlated electron systems whereas the 122 pnictides are considered as weakly correlated one but showing similar effects). In general, the Lifshitz transition is found in some of the 122 family compounds that are grown by various experimental (other than Sn flux) methods [36]. However, besides the hole or electron doping effects, the localization effects from impurity scattering may significantly affect the Fermi surface as well [37] and the contamination effect by impurity is not restricted to Sn-flux method only (see for example occurence of Lifshitz transition as an effect of $\mathrm{Ca}$ impurities being unintentionally present in fcc- $\mathrm{Yb}$ crystals [38]). Therefore, our study is of general significance.

Single crystals of $\mathrm{BaFe}_{2} \mathrm{As}_{2}$ (Ba122), $\mathrm{SrFe}_{2} \mathrm{As}_{2}$ ( $\left.\mathrm{Sr} 122\right)$ and $\mathrm{CaFe}_{2} \mathrm{As}_{2}$ (Ca122), synthesized from tin-flux method contain a few percentage (1\%$5 \%$ ) of Sn incorporated into the crystal structure. As a result of Sn impurity, the crystallographic structure of 122 systems get modified [31, 39, 40]. It was found that $95 \%$ of the 2 a site $(0,0,0)$ is occupied by Ba atoms and the rest are found to be replaced by $\mathrm{Sn}$ atoms at the site $4 \mathrm{e}(0,0, z$ with $z=0.093)$ [40]. It is obvious from current literature that electronic structures of 122 systems are highly influenced by certain moderation of structural parameters [22, 26, 41]. This incorporation of Sn impurity leads to certain changes in the 
physical properties of Ba122 system. The presence of a small fraction of Sn impurity in $\mathrm{BaFe}_{2} \mathrm{As}_{2}$ (Ba122) system curtails down the structural as well as SDW transitions from $138 \mathrm{~K}$ to $85 \mathrm{~K}$ and also give rise to a notable difference in the thermal behaviour of electrical resistivity and magnetic susceptibility $[31,42,43,44]$. On the other hand, no significant modifications in the physical properties of Sr122 and Ca122 systems are observed due to the presence of Sn impurity. Since these structural and magnetic transitions are intimately related to the electronic structures, it calls for the study of detailed electronic structure through density of states (DOS), band structures and Fermi surfaces of $\mathrm{BaFe}_{2} \mathrm{As}_{2}$ in presence of Sn impurity. Such study is absent from current literature. Within virtual crystal approximation, we have made the first attempt to explain the observed diversities in the physical properties of these 122 materials due to Sn impurity. Our theoretical investigation using first principles density functional theory reveals that in Ba122 system, an impurity induced Lifshitz transition occurs but such transition is absent in other 122 systems like Sr122 and Ca122 systems. This may be the reason for observed differences in the physical properties of various 122 materials. Our manifestation of Sn impurity in the crystal structures through VCA method, slightly deviates from the ideal experimental conditions. We discuss this issue in the theoretical method section along with the details of calculations. In the next section, we present our detail calculated DOS, band structures and FSs of Ba122, Sr122 and Ca122 systems with inbuilt Sn impurity. In the last section we present the summary of our work with conclusions.

\section{Theoretical method}

Our first principles electronic structure calculations are performed by using CASTEP which is a plane wave psudopotential method based on density functional theory [45], where the electronic exchange correlation is treated under the generalized gradient approximation (GGA) using Perdew-BurkeErnzerhof (PBE) functional [46]. Experimentally measured orthorhombic $(20 \mathrm{~K})$ as well as tetragonal $(300 \mathrm{~K})$ structural parameters i.e., $a, b, c$ and $z_{A s}$ (fractional z co-ordinate of As atom) [39, 40, 47, 48] are used as inputs of our first principles density of states (DOS), band structures and Fermi surface calculations. Implementation of $\mathrm{Sn}$ impurity in $\mathrm{BaFe}_{2} \mathrm{As}_{2}$ and $\mathrm{SrFe}_{2} \mathrm{As}_{2}$ systems has been treated within virtual crystal approximation. Virtual crystal approximation is an well known method for treating disordered systems within primitive unit cell [49]. This VCA method forges us to use the same 
atomic co-ordinates for $\mathrm{Ba}$ and $\mathrm{Sn}$ atoms which is not the actual condition where Sn atoms are situated at slightly different co-ordinates $(0,0,0.093)$ close to $\mathrm{Ba}(0,0,0)$ atom. (In general, Sn shows a chemical similarity to both neighbouring group-14 elements, germanium and lead, and has two possible oxidation states, +2 and slightly more stable +4 .) This displacement of Sn atom however, is accounted for the stereo-chemical active lone pair of the formal divalent $\mathrm{Sn}$ atom that results in a change of the coordination sphere which is a $\mathrm{Sn}^{2+}$-typical square pyramidal coordination by four As atoms [40]. In this work, we use the virtual crystal approximation (VCA) method, developed by Bellaiche and Vanderbilt [49] based on weighted averaging of pseudopotentials. In VCA, a doped crystal with original periodicity but composed of fictitious 'virtual atoms' is created to mimic the actual doped system. For example, one can construct a virtual atom like $\mathrm{A}_{1-x} \mathrm{~B}_{x}$ for a single lattice site, where $x$ is the doping concentration and thus overlooks any possible short range order. One uses ultra-soft pseudopotentials which are prone to generating ghost states in the VCA framework, while none of the individual potentials has a ghost state. There is a standard way of testing the VCA approach for ghost states, so one should always examine the value of the derivative of the total energy w.r.t. $\mathrm{E}_{c u t}\left(\mathrm{E}_{c u t}\right.$ defines the size of plane wave basis set) during the finite basis set correction calculation. It should be of the same order as the derivatives for the end-member structures [50]. We have checked this value for all these three systems up to 5\% Sn doping in order to inspect the applicability of VCA method in these cases. Both spinpolarized (for orthorhombic phase with space group symmetry Fmmm [No. 69]) as well as non-spin-polarized single point energy calculations are carried out (for tetragonal phase with space group symmetry I4/mmm [No. 139]) using ultrasoft pseudo-potentials and plane-wave basis set with energy cut off $600 \mathrm{eV}$ and self-consistent field (SCF) tolerance $10^{-6} \mathrm{eV} /$ atom. Brillouin zone is sampled in the $\mathrm{k}$ space within MonkhorstPack scheme and grid size for SCF calculation is chosen as $26 \times 26 \times 26$.

\section{Results and discussion}

Our main aim is to examine effect of Sn impurity in the electronic structures of Ba122, Sr122 and Ca122 systems within VCA method. We calculate DOS, band structures and FSs of these three systems (pure and Sn contaminated) using experimental lattice parameters $\left(a, b, c, \mathrm{z}_{A s}\right)$ in the orthorhombic (low temperature) phases. No significant differences in the calculated 
DOS for pure and Sn contaminated 122 systems are observed. We exhibit our calculated electronic structure in the orthorhombic phase where experimentally measured orthorhombic lattice parameters are used as the input of our first principles calculations. It should be mentioned here that the structural transition in these materials is due to the orbital ordering between $\mathrm{d}_{y z}$ and $\mathrm{d}_{x z}$ bands around high symmetry $\mathrm{k}$ points [6]. In FIG.1 we depict the FSs of $\mathrm{BaFe}_{2} \mathrm{As}_{2}$ systems with various percentages of $\mathrm{Sn}$ impurity. There are two electron like FSs around the four corners and three hole like FSs at the centre of the Brillouin Zone (BZ). In order to provide a clearer view of all the FSs, we present the FSs separately in second and third rows of FIG.1. One can see from these FSs that with increasing Sn impurity electron like FSs shrink and hole like FSs expand, which corroborates with the previous experimental picture of hole doping as a consequence of Sn impurity in Ba122 structure [43]. One of our most important observations, is the occurrence of Lifshitz transition upon certain percentage of Sn impurity (see FIG.1 and FIG. 2). With 4\% Sn impurity in Ba122 system, the innermost FS at the centre of the BZ vanishes around $\Gamma$ point as a direct consequence of Lifshitz transition.

On the whole with increasing Sn percentage, the shapes of FSs become more 3D like, resulting in large degradation of FS nesting. As a result of degradation of nesting magnetic order becomes weaker which might be the reason of lowering of structural as well as SDW transition temperatures $\left(\mathrm{T}_{s}\right.$ and $\left.\mathrm{T}_{S D W}\right)$ in Sn contaminated Ba122 systems. To illustrate the observed Lifshitz transition, we exhibit the calculated band structures of Sn contaminated Ba122 system for 2\%, 4\% and 5\% Sn impurity in FIG 3 . Band structures around $\Gamma, \mathrm{X}$ and $\mathrm{Y}$ points are shown in first, second and third rows of FIG 3 respectively. It is quite evident from these figures that at around 4\% Sn impurity Lifshitz transition occur in Ba122 system. As a signature of this Lifshitz transition, a hole like band $\left(\mathrm{d}_{x y}\right.$ band around $\Gamma$ point) becomes electron like (see FIG $3 \mathrm{~b}$ ). Observation of Lifshitz transition in other high symmetry k points also occur as clearly demonstrated in FIG 3. This type of transition can create remarkable topological modification of FSs, which might be the reason of alteration of superconducting properties of these Ba122 systems with Sn impurity. Transition of hole like band to electron like band as well as disappearance of electron like band from Fermi level (FL) would not only modify the shape of the FSs but also affect the nesting condition between electron and hole like bands severely. Since nesting of FS is directly related to magnetic SDW transition, lowering of observed magnetic transi- 


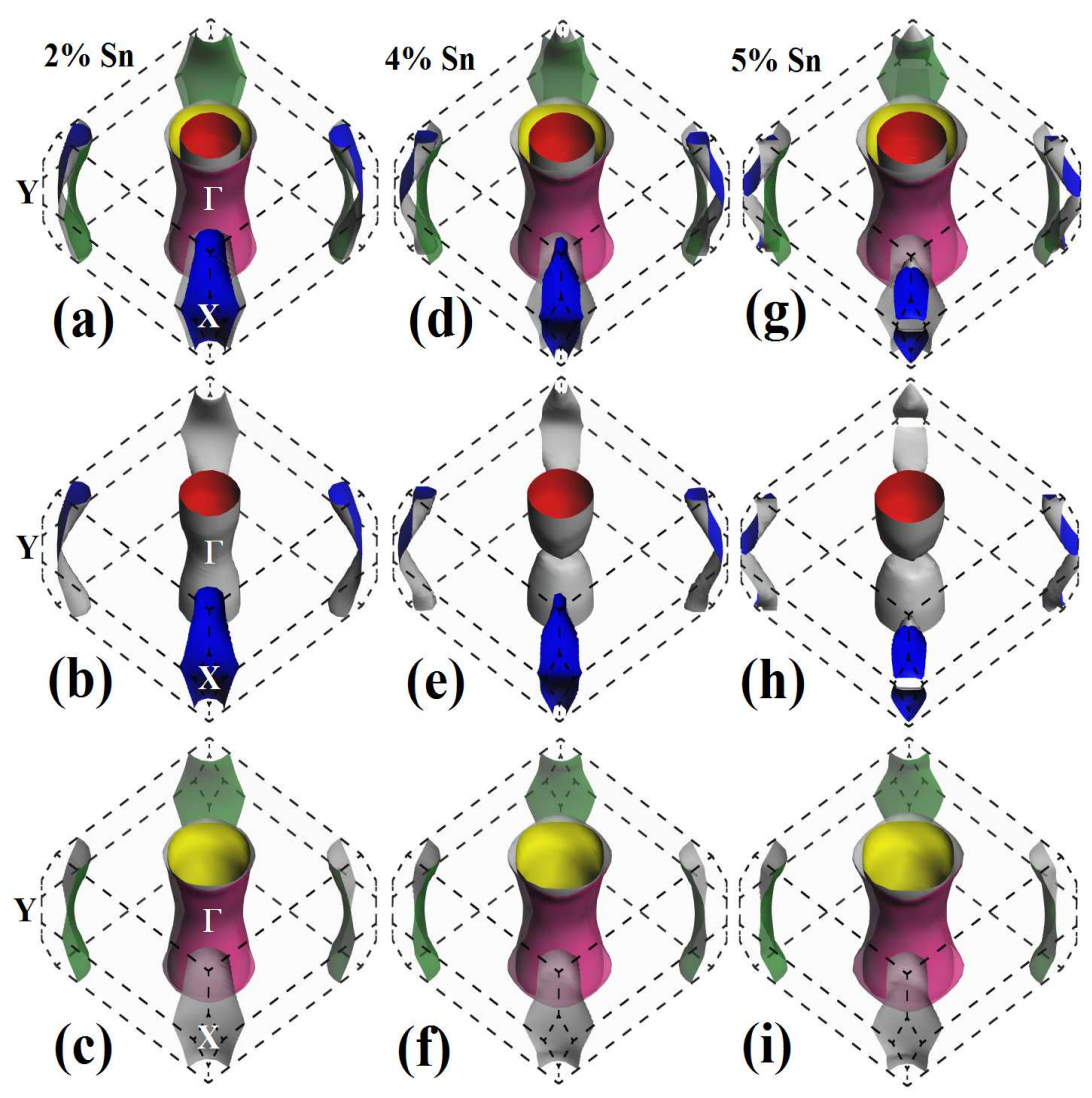

Figure 1: Calculated Fermi surfaces in orthorhombic phase within VCA method for (a, b, c) $\mathrm{Ba}_{0.98} \mathrm{Sn}_{0.02} \mathrm{Fe}_{2} \mathrm{As}_{2}$, (d, e, f) $\mathrm{Ba}_{0.96} \mathrm{Sn}_{0.04} \mathrm{Fe}_{2} \mathrm{As}_{2}$ and (g, h, i) $\mathrm{Ba}_{0.95} \mathrm{Sn}_{0.05} \mathrm{Fe}_{2} \mathrm{As}_{2}$ systems. For clarity individual FSs are shown separately in 2nd and 3rd row. Different colours are used to indicate different FSs. $\mathrm{k}$ points in the Brillouin Zone (BZ) are also indicated in (a). Shrinkage of electron like FSs and surging of hole like FSs are worth noticing. This indicates hole doping. 

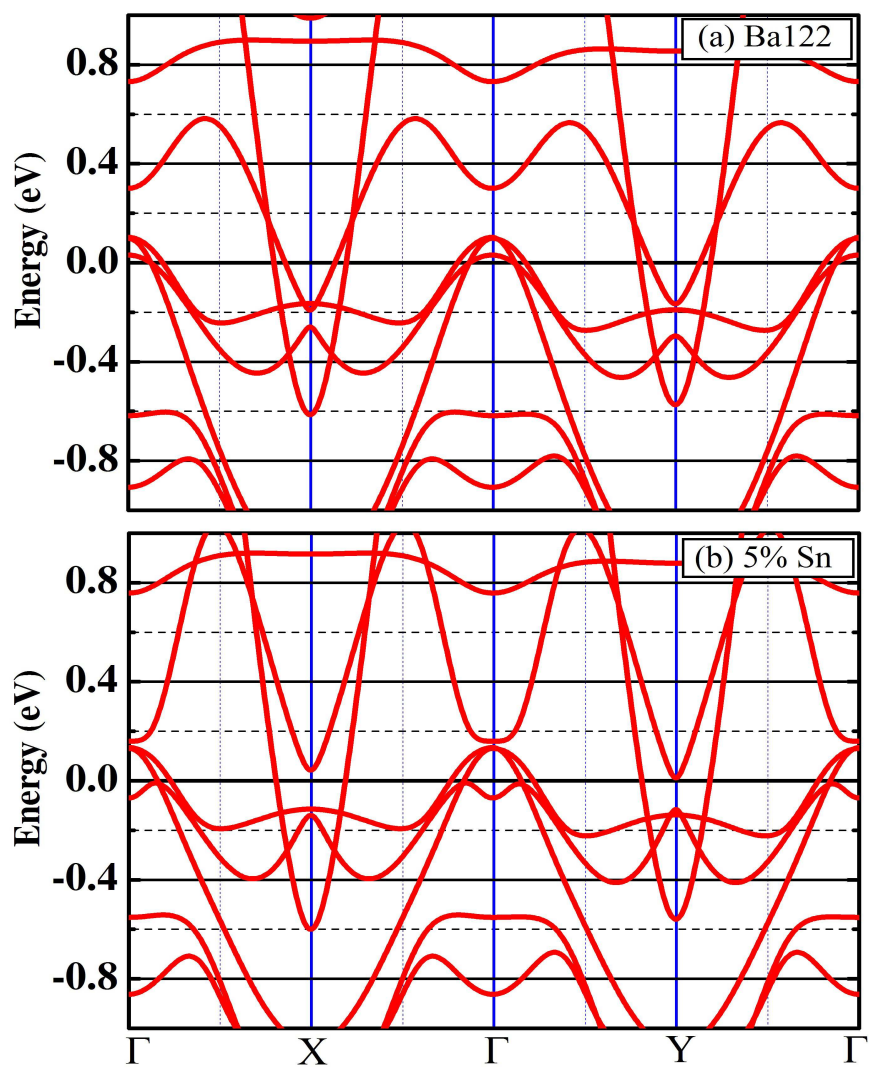

Figure 2: Calculated band structures in orthorhombic phase within VCA method for (a) $\mathrm{BaFe}_{2} \mathrm{As}_{2}$ and (b) $\mathrm{Ba}_{0.95} \mathrm{Sn}_{0.05} \mathrm{Fe}_{2} \mathrm{As}_{2}$ systems along $\Gamma-X-\Gamma-Y-\Gamma$ k-path in the BZ. In comparison to FIG 5, 5\% Sn doped Ba122 system shows significant modification in band structure as far as electronic topological transitions are concerned. 

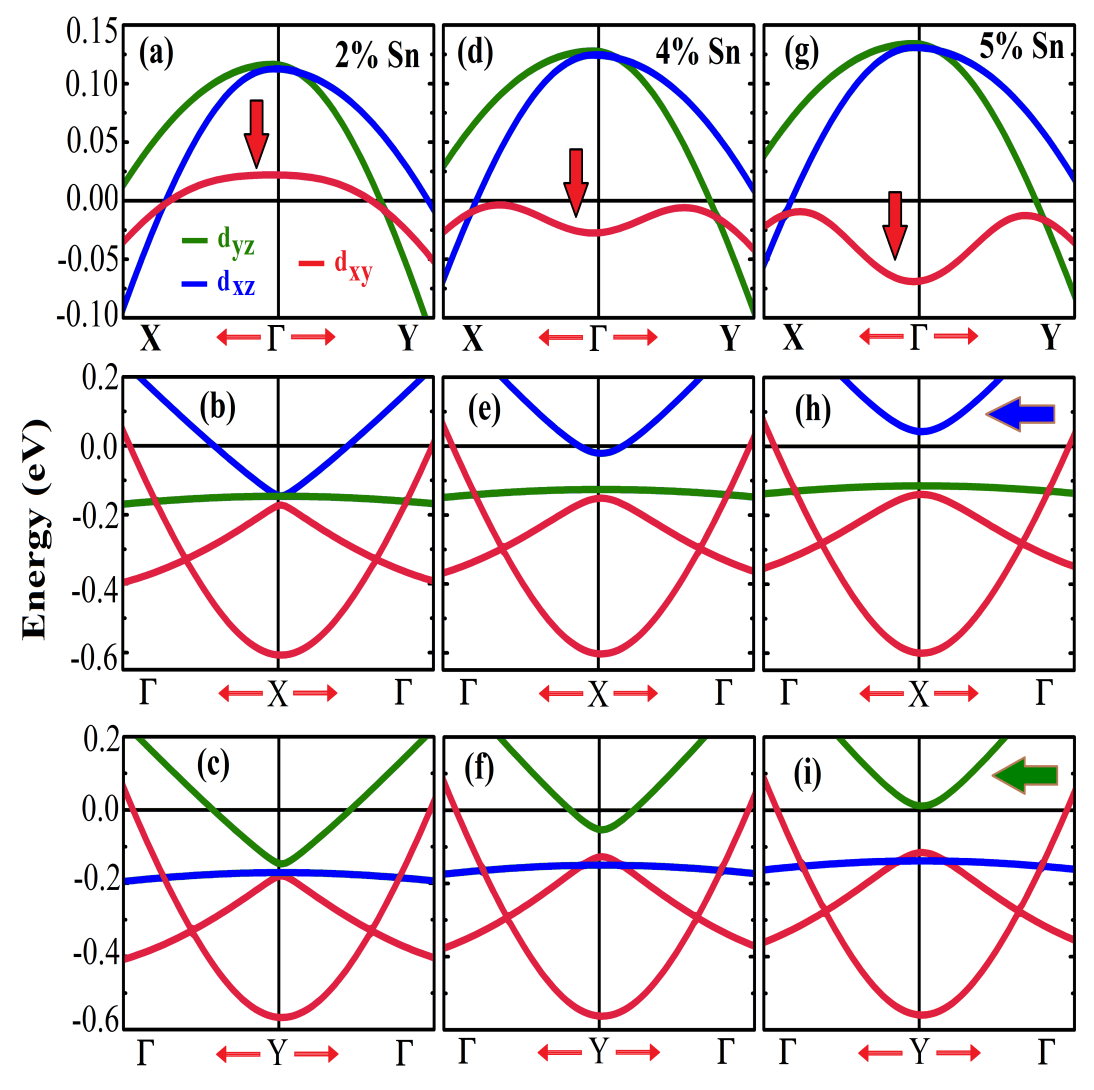

Figure 3: Calculated band structures in orthorhombic phase within VCA method for (a, b, c) $\mathrm{Ba}_{0.98} \mathrm{Sn}_{0.02} \mathrm{Fe}_{2} \mathrm{As}_{2}$, (d, e, f) $\mathrm{Ba}_{0.96} \mathrm{Sn}_{0.04} \mathrm{Fe}_{2} \mathrm{As}_{2}$ and (g, h, i) $\mathrm{Ba}_{0.95} \mathrm{Sn}_{0.05} \mathrm{Fe}_{2} \mathrm{As}_{2}$ systems around $\Gamma$ (1st row), X (2nd row) and Y (3rd row) points. Electronic topological transition (transition of hole like band to electron like band) is indicated using red arrow around $\Gamma$ point. Movement of the $\mathrm{d}_{x z}\left(\mathrm{~d}_{y z}\right)$ band around $\mathrm{X}(\mathrm{Y})$ point away from the Fermi level is shown by blue (green) arrow. This figure may be contrasted with FIG 6 and FIG 9 


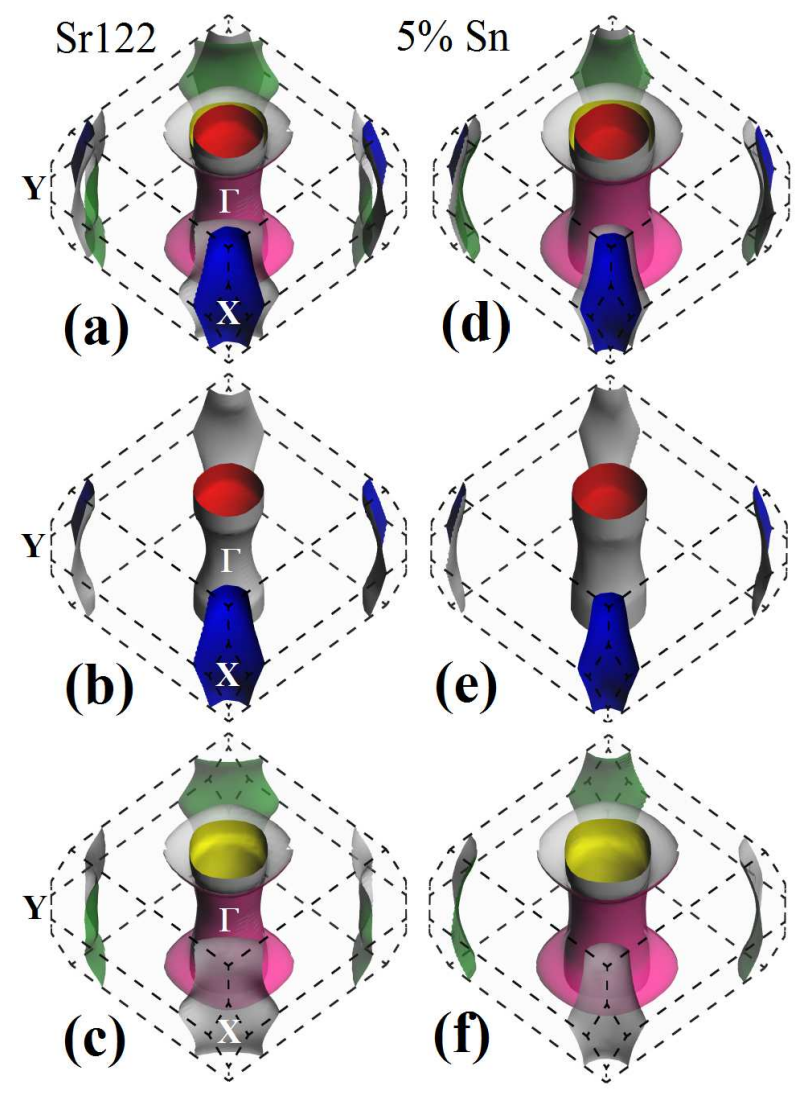

Figure 4: Calculated Fermi surfaces in orthorhombic phase within VCA method for (a, b, c) $\mathrm{SrFe}_{2} \mathrm{As}_{2}$ and (d, e, f) $\mathrm{Sr}_{0.95} \mathrm{Sn}_{0.05} \mathrm{Fe}_{2} \mathrm{As}_{2}$ systems. For clarity individual FSs are shown separately in 2nd and 3rd row. Shrinkage of electron like FSs and surging of hole like FSs are worth noticing. This indicates hole doping. 


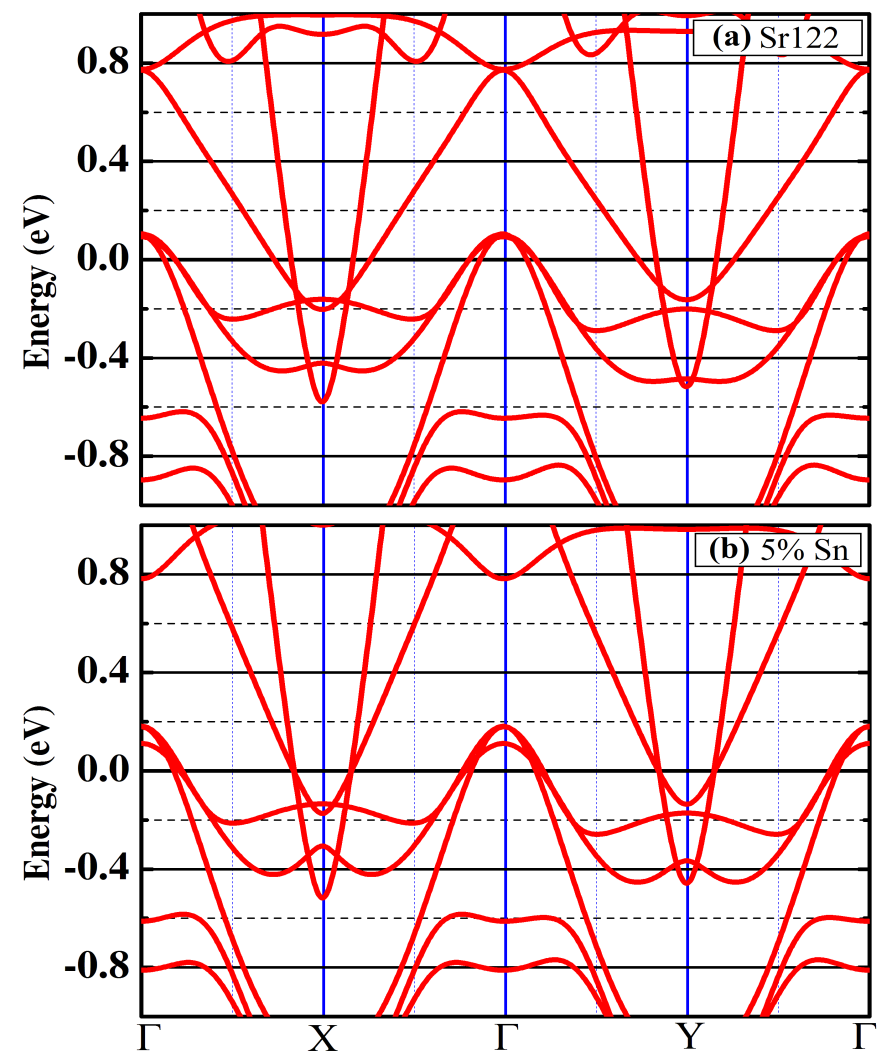

Figure 5: Calculated band structures in orthorhombic phase within VCA method for (a) $\mathrm{SrFe}_{2} \mathrm{As}_{2}$ and (b) $\mathrm{Sr}_{0.95} \mathrm{Sn}_{0.05} \mathrm{Fe}_{2} \mathrm{As}_{2}$ systems along $\Gamma-X-\Gamma-Y-\Gamma$ k-path in the BZ. In comparison to FIG 2, 5\% Sn doped Sr122 system shows insignificant modification in band structure as far as electronic topological transitions are concerned. 
tion in Sn contaminated Ba122 system may be attributed to the electronic topological transition as found from our first principles simulations. Band structures around $\mathrm{X}$ and $\mathrm{Y}$ points reveal that orbital order also got modified in the presence of slightly higher percentages of Sn impurities $(>1 \%)$. This might stand out as an explanation of reduction of structural transition temperature $\left(\mathrm{T}_{s}\right)$. On the other hand, there is no experimental evidence of lowering structural transition temperature $\left(\mathrm{T}_{s}\right)$ and SDW transition temperature $\left(\mathrm{T}_{S D W}\right)$ in Sr122 and Ca122 compounds, prepared by Sn-flux method (containing Sn impurity). We have also performed electronic structure calculation of Sn contaminated Sr122 and Ca122 systems. In FIG 4 we have displayed the FSs of Sr122 system (first column) as well as 5\% Sn contaminated Sr122 system (second column). In FIG.77 we present the FSs of Ca122 system (first column) as well as 5\% Sn contaminated Ca122 system (second column). Calculated FSs of Sr122 and Ca122 system with 5\% Sn impurity (FIG 4 and FIG 7) give a clear indication of hole doping i.e., expansion of hole FSs and shrinkage of electron FSs. However, there is no trace of Lifshitz transition in case of Sn contaminated Sr122 and Ca122 systems contrary to the case of Ba122 system. Besides there is no significant change in the FS topology specially, the change in dimensionality of FSs in presence of $5 \% \mathrm{Sn}$ impurity. So there is no evidence of degradation of FS nesting in these two systems. This may also stand out as an explanation for the robustness of magnetic, structural and superconducting transition temperatures for Sr122 and Ca122 systems with Sn impurity. We have also simulated the band structure of Sr122 and Ca122 systems in order to see the moderation of orbital order if any. In FIG $5 \mathrm{~F}$, the band structure along $\mathrm{k}$ path $\Gamma-X-\Gamma-Y$ of pure Sr122 system and in FIG 5 b that for 5\% Sn contaminated Sr122 systems are presented. Similarly in FIG 8 a, the band structure along k path $\Gamma-X-\Gamma-Y$ of pure Ca122 system and in FIG 8 b that for $5 \%$ Sn contaminated Ca122 systems are depicted. Band structures of these pure and impure systems have no qualitative differences. In FIG 6 and FIG 9 band structure around $\Gamma, \mathrm{X}$ and Y points of pure as well as 5\% Sn contaminated Sr122 and Ca122 systems have been shown. There is no sign of Lifshitz transition or any kind of electronic topological transition (ETT) at least up to 5\% Sn impurity. And there is almost no change in the nature of orbital ordering between $\mathrm{d}_{y z}$ and $\mathrm{d}_{x z}$ bands around $\Gamma, \mathrm{X}$ and $\mathrm{Y}$ point with the introduction of Sn impurity (5\% Sn) in Sr122 and Ca122 systems. Since orbital ordering of $\mathrm{d}_{y z}$ and $\mathrm{d}_{x z}$ bands around $\mathrm{X}$ and $\mathrm{Y}$ points are the major contributors to the structural transition from tetragonal (high temperature) to orthorhombic 

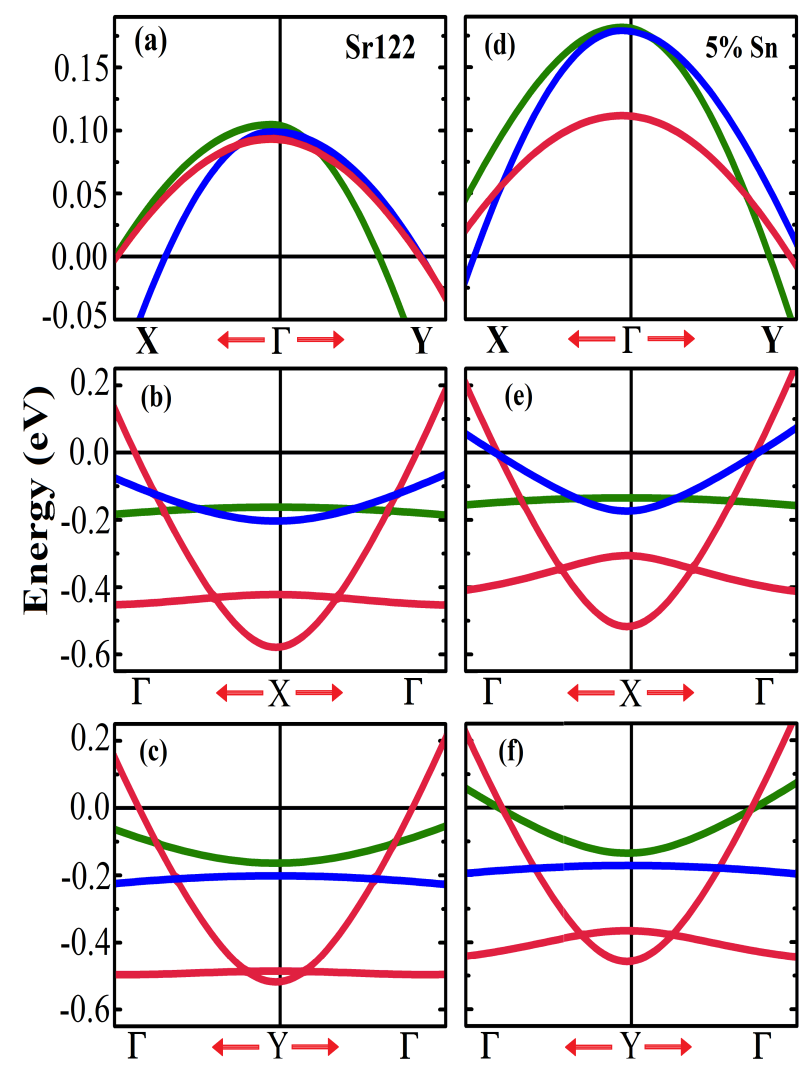

Figure 6: Calculated band structures in orthorhombic phase within VCA method for (a, b, c) $\mathrm{SrFe}_{2} \mathrm{As}_{2}$ and (d, e, f) $\mathrm{Sr}_{0.95} \mathrm{Sn}_{0.05} \mathrm{Fe}_{2} \mathrm{As}_{2}$ systems around $\Gamma$ (1st row), X (2nd row) and Y (3rd row) points. Modification in the band widths of electron (reduction in band width) and hole (increment in band width) like bands causes apprehendable modifications in the FSs (See FIG 4). 
phase (low temperature) in 122 systems [12, 6], no significant moderation of orbital ordering around X and Y points support the experimental observation of the unaffected structural transition temperature in Sn induced Sr122 and Ca122 systems. Lifshitz transition with variation of pressure or doping concentration or even with variation of temperature, plays an important role in 122 Fe-based superconductors and is observed experimentally [27, 28]. Thus one must be sure about the fact whether the observed Lifshitz transitions are artefacts of Sn impurity or not. So by using VCA method for introducing Sn into the Ba122/Sr122/Ca122 structure, we provide a detailed electronic structures for pure and Sn contaminated systems for the first time. We also elucidate the experimentally observed anomalies for these Sn induced 122 systems through our first principles electronic structure calculation which is not available in the current literature.

In order to understand the influence of Sn doping on various 122 materials it is essential to identify that the band structures of undoped Ba122, Sr122 and Ca122 systems (in the orthorhombic phase) already have significant differences. For Ba122 system, electronic bands around $\Gamma$ point is very close to the Fermi level (FL), specially, the flat $\mathrm{d}_{x y}$ band nearly touches the FL. The other two bands $\mathrm{d}_{x z}$ and $\mathrm{d}_{y z}$ are slightly higher in energy compared to that of the $\mathrm{d}_{x y}$. This should be contrasted with that of the Sr122 and Ca122 systems (see FIG,2, 5, 8), where all the $\mathrm{d}_{x y}, \mathrm{~d}_{x z}$ and $\mathrm{d}_{y z}$ ) bands are further away from FL. This is because of the largest size of $\mathrm{Ba}(253 \mathrm{pm})$ compared to that of $\mathrm{Sr}(219 \mathrm{pm})$ and $\mathrm{Ca}(194 \mathrm{pm})$ causing two Fe-As layers furthest in Ba122 compared to Sr122, Ca122. As a result Ba-122 is more planer compared to others (see for example, a dimensional crossover of FS in 122 systems [41] ) resulting a flatter $\mathrm{d}_{x y}$ band near FL It is to be noted that the $\mathrm{d}_{x y}$ band is planar $(x y)$ in nature in contrast to the $\mathrm{d}_{x z} / \mathrm{d}_{y z}$ bands. Sn substitution in the M-atoms $(\mathrm{Ba} / \mathrm{Sr} / \mathrm{Ca})$ is an out-of-plane substitution. Ba atom being the largest in size among the M-atoms as well as Sn, a few percentage of Sn substitution keeps the effective size of the 'virtual M atom' largest in size in case of $\mathrm{Ba}$, smaller in case of $\mathrm{Sr}$ and the smallest in case of Ca. This would cause vertical movement between the Fe-As planes least for Sn-doped Ba-122 but larger for Sn-doped Sr-122 and largest for Sn-doped Ca-122. Therefore, naturally $\mathrm{d}_{x z} / \mathrm{d}_{y z}$ bands would move furthest in case of Sn-doped Ca-122 whereas least movement to the $\mathrm{d}_{x y}$ bands (see for example, Figures 3, 6, 9). That also simultaneously explains hole doping effect. Thus, Sn impurity causes splitting between the $\mathrm{d}_{x y}$ and the pair of "nearly 'degenerate bands $\mathrm{d}_{x z} / \mathrm{d}_{y z}$. The modulations of the $\mathrm{d}_{x z} / \mathrm{d}_{y z}$ bands can also 


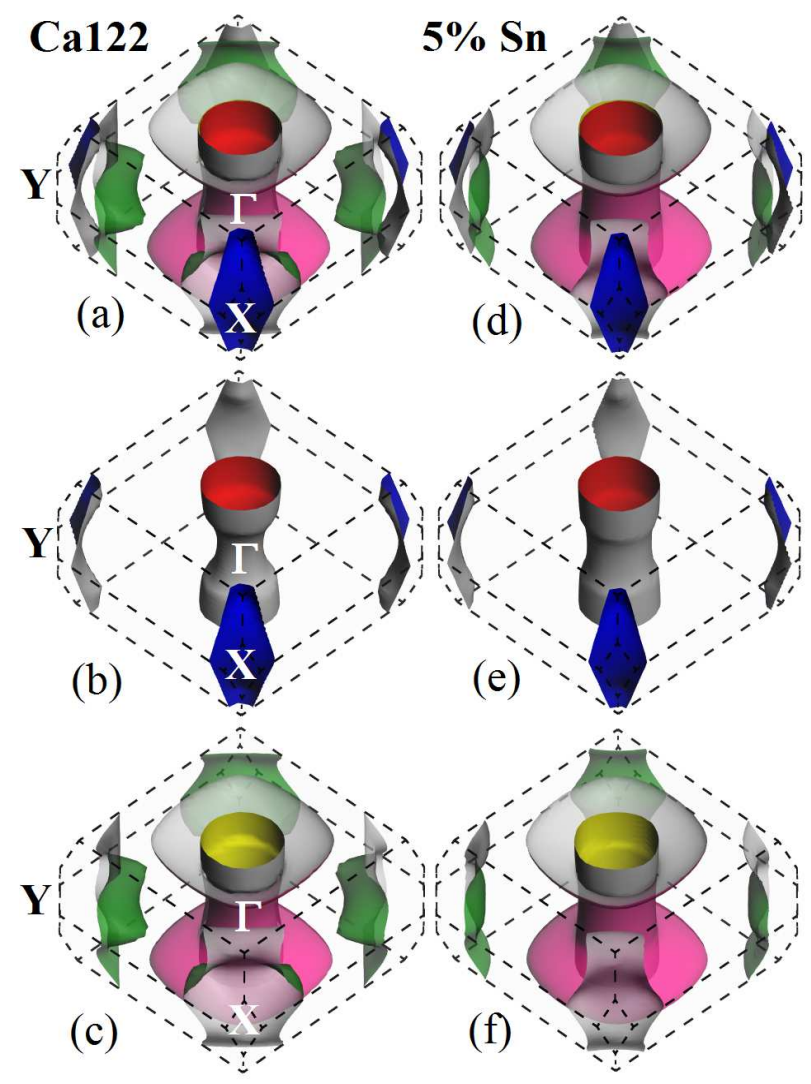

Figure 7: Calculated Fermi surfaces in orthorhombic phase within VCA method for (a, b, c) $\mathrm{CaFe}_{2} \mathrm{As}_{2}$ and (d, e, f) $\mathrm{Ca}_{0.95} \mathrm{Sn}_{0.05} \mathrm{Fe}_{2} \mathrm{As}_{2}$ systems. For clarity individual FSs are shown separately in 2nd and 3rd row. Shrinkage of electron like FSs and surging of hole like FSs are worth noticing. This indicates hole doping. 


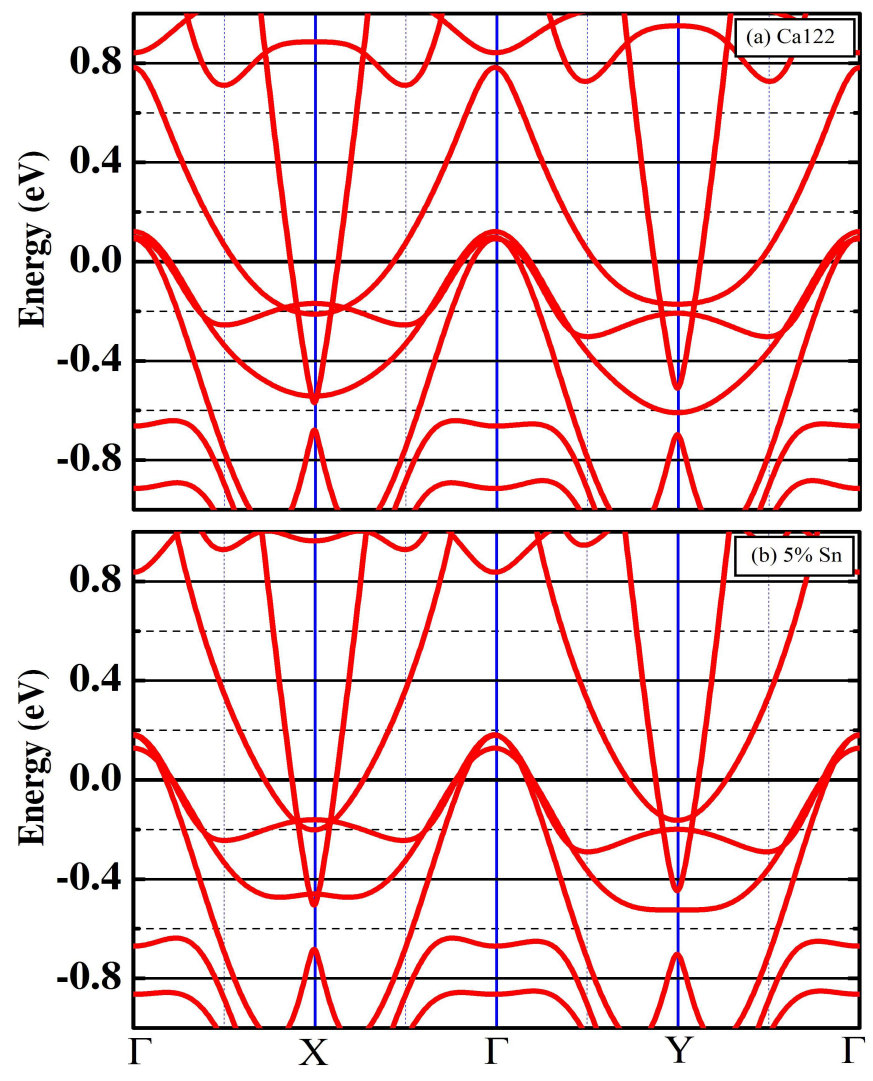

Figure 8: Calculated band structures in orthorhombic phase within VCA method for (a) $\mathrm{CaFe}_{2} \mathrm{As}_{2}$ and (b) $\mathrm{Ca}_{0.95} \mathrm{Sn}_{0.05} \mathrm{Fe}_{2} \mathrm{As}_{2}$ systems along $\Gamma-X-\Gamma-Y-\Gamma$ k-path in the BZ. In comparison to FIG 2, 5\% Sn doped Ca122 system shows insignificant modification in band structure as far as electronic topological transitions are concerned. 

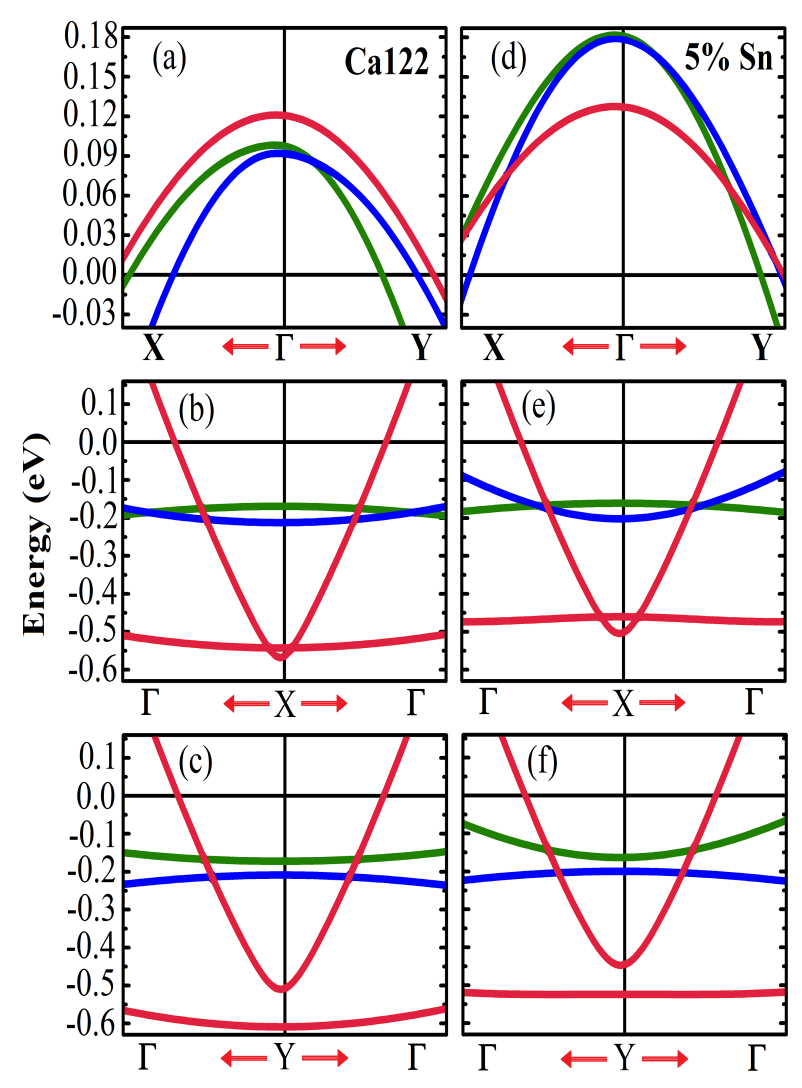

Figure 9: Calculated band structures in orthorhombic phase within VCA method for (a, b, c) $\mathrm{CaFe}_{2} \mathrm{As}_{2}$ and (d, e, f) $\mathrm{Ca}_{0.95} \mathrm{Sn}_{0.05} \mathrm{Fe}_{2} \mathrm{As}_{2}$ systems around $\Gamma$ (1st row), $\mathrm{X}$ (2nd row) and Y (3rd row) points. Modification in the band widths of electron (reduction in band width) and hole (increment in band width) like bands causes apprehendable modifications in the FSs (See FIG 7). 
produce modulations in the $\mathrm{d}_{x y}$ bands. But the $\mathrm{d}_{x y}$ band around the $\Gamma$ point is much lower in energy compared to that of the $\mathrm{d}_{x z} / \mathrm{d}_{y z}$ bands and closer to the FL for the undoped Ba122. This is not the case of undoped Sr122, Ca122 where $\mathrm{d}_{x y} / \mathrm{d}_{x z} / \mathrm{d}_{y z}$ are nearly at same but higher in energy. The effect of modulations in the $\mathrm{d}_{x y}$ bands due to that of the $\mathrm{d}_{x z} / \mathrm{d}_{y z}$ bands is thus minimum in case of $\mathrm{Sr}-122, \mathrm{Ca}-122$ systems. The consequence of $\mathrm{Sn}$ impurity on 122 family materials thus depend on strong doping sensitivity of the underlying system - energy levels of $\mathrm{d}_{x y} / \mathrm{d}_{x z} / \mathrm{d}_{y z}$ and the impurity energy level. The $\mathrm{d}_{x y}$ band of Ba122 being already very close to the FL in presence

of Sn impurity is pushed down below the FL due to such modulation, causing the Lifshitz transition.

\section{Conclusion}

We have studied the effect of $\mathrm{Sn}$ impurity on the electronic structures of 122 systems $\left(\mathrm{BaFe}_{2} \mathrm{As}_{2}, \mathrm{SrFe}_{2} \mathrm{As}_{2}\right.$ and $\left.\mathrm{CaFe}_{2} \mathrm{As}_{2}\right)$ using VCA method for introducing Sn impurity into the 122 crystal structures. Presence of Sn impurity modifies the electronic structures of Ba122 system remarkably, which in turn is expected to result certain changes in the physical properties like reduction of structural and magnetic transition temperatures as well as superconducting transition temperature. On the contrary, Sn impurity does not have much impact on the electronic structures of Sr122 and Ca122 systems as reflected in the experimental evidence of robustness of structural, magnetic and superconducting transition temperatures [31, 51]. In Ba122 system, we observe Sn impurity induced Lifshitz transition around $\Gamma$ point that dictates several changes in the electronic structures near Fermi level including degradation of FS nesting contrasting the case of Sr122 and Ca122 systems. Orbital ordering between $\mathrm{d}_{y z}$ and $\mathrm{d}_{x z}$ bands around $\mathrm{X}$ and $\mathrm{Y}$ points, which is responsible for structural transition, remains unaltered in case of Sr122 and Ca122 systems but is substantially modified in case of Ba122 system in presence of Sn impurity. This work thus not only provide a detailed description of electronic structures of Sn contaminated 122 systems but also provides a possible explanation to the various observed anomalies in the physical properties that are not well understood so far. We believe our work will lead to further similar research beyond VCA. 


\section{Acknowledgments}

One of us (SS) acknowledges the HBNI, RRCAT for financial support and encouragements. We thank Dr. P. A. Naik and Dr. P. D. Gupta for their encouragement in this work.

\section{References}

[1] Clarina de la Cruz et al., Magnetic order close to superconductivity in the iron-based layered $\mathrm{LaO}_{1-x} \mathrm{~F}_{x}$ FeAs systems, Nature 453 (2008) 899.

[2] J. Dong, H. J. Zhang, G. Xu, Z. Li, G. Li, W. Z. Hu, D. Wu, G. F. Chen, X. Dai, J. L. Luo, Competing orders and spin-density-wave instability in La $\left(\mathrm{O}_{1-x} \mathrm{~F}_{x}\right)$ FeAs, Europhys. Lett. 83 (2008) 27006.

[3] S. Avci et al., Magnetically driven suppression of nematic order in an iron-based superconductor, Nat. Commun. 5 (2014) 3845.

[4] T. Shimojima et al., Orbital-Dependent Modifications of Electronic Structure across the Magnetostructural Transition in $\mathrm{BaFe}_{2} \mathrm{As}_{2}$, Phys. Rev. Lett. 104 (2010) 057002.

[5] C.-C. Chen et al., Orbital order and spontaneous orthorhombicity in iron pnictides, Phys. Rev. B 82 (2010) 100504(R).

[6] Smritijit Sen, Haranath Ghosh, A. K. Sinha and A. Bharathi, Origin of structural and magnetic transitions in $\mathrm{BaFe}_{2-x} \mathrm{Ru}_{x} \mathrm{As}_{2}$ materials, $\mathrm{Su}-$ percond. Sci. Technol.27 (2014) 122003.

[7] Haranath Ghosh and Harsh Purwar, Elementary and collective excitations as probes for order parameter symmetry in Fe-based superconductors, Europhys. Lett. 98 (2012) 57012.

[8] Kalyan Sasmal et al., Superconducting Fe-Based Compounds $\left(\mathrm{A}_{1-x} \mathrm{Sr}_{x}\right) \mathrm{Fe}_{2} \mathrm{As}_{2}$ with $\mathrm{A}=\mathrm{K}$ and $\mathrm{Cs}$ with Transition Temperatures up to 37 K, Phys. Rev. Lett. 101 (2008) 107007.

[9] Q. Huang et al., Neutron-Diffraction Measurements of Magnetic Order and a Structural Transition in the Parent $\mathrm{BaFe}_{2} \mathrm{As}_{2}$ Compound of FeAsBased High-Temperature Superconductors, Phys. Rev. Lett. 101 (2008) 257003. 
[10] S.-H. Baek et al., Orbital-driven nematicity in FeSe, Nature Mater. 14 (2015) 210.

[11] R. M. Fernandes et al., What drives nematic order in iron-based superconductors, Nature Phys. 10 (2014) 97.

[12] Smritijit Sen and Haranath Ghosh, Nematicity, magnetic fluctuation and ferro-spin-orbital ordering in $\mathrm{BaFe}_{2} \mathrm{As}_{2}$ family, J. Alloys Compd. doi:10.1016/j.jallcom.2016.03.034 (2015).

[13] Awadhesh Mani et al., Pressure-induced superconductivity in BaFe2As2 single crystal, Europhys. Lett. 87 (2009) 17004.

[14] Tuson Park et al., Pressure-induced superconductivity in $\mathrm{CaFe}_{2} \mathrm{As}_{2}$, J. Phys.: Condens. Matter. 20 (2008) 322204.

[15] A. S. Sefat et al., Superconductivity at $22 \mathrm{~K}$ in Co-Doped $\mathrm{BaFe}_{2} \mathrm{As}_{2}$ Crystals, Phys. Rev. Lett. 101 (2008) 117004.

[16] A. Leithe-Jasper et al., Superconducting State in $\mathrm{SrFe}_{2-x} \mathrm{Co}_{x} \mathrm{As}_{2}$ by Internal Doping of the Iron Arsenide Layers, Phys. Rev. Lett. 101 (2008) 207004.

[17] H. Kontani and S. Onari, Orbital-Fluctuation-Mediated Superconductivity in Iron Pnictides: Analysis of the Five-Orbital Hubbard-Holstein Model, Phys. Rev. Lett. 104 (2010) 157001.

[18] I. Mazin et al., Unconventional Superconductivity with a Sign Reversal in the Order Parameter of $\mathrm{LaFeAsO}_{1 x} \mathrm{~F}_{x}$, Phys. Rev. Lett. 101 (2008) 057003.

[19] A. V. Chubukov, Pairing mechanism in Fe-based superconductors, Annul. Rev. Cond. Mat. Phys. 3 (2012) 13.1.

[20] S. Onari and H. Kontani, Self-consistent Vertex Correction Analysis for Iron-based Superconductors: Mechanism of Coulomb Interaction-Driven Orbital Fluctuations, Phys. Rev. Lett. 109 (2012) 137001.

[21] G. R. Stewart, Superconductivity in iron compounds, Rev. Mod. Phys. 83 (2011) 1589. 
[22] D. Kasinathan et al., $\mathrm{AFe}_{2} \mathrm{As}_{2}(\mathrm{~A}=\mathrm{Ca}, \mathrm{Sr}, \mathrm{Ba}, \mathrm{Eu})$ and $\mathrm{SrFe}_{2-x} \mathrm{TM}_{x} \mathrm{As}_{2}$ $(\mathrm{TM}=\mathrm{Mn}, \mathrm{Co}, \mathrm{Ni})$ : crystal structure, charge doping, magnetism and superconductivity, New J. Phys. 11 (2009) 025023.

[23] Smritijit Sen and Haranath Ghosh, Intra-inter band pairing, order parameter symmetry in Fe-based superconductors: A model study, J. Alloys Compd. 618 (2015) 102.

[24] Smritijit Sen and Haranath Ghosh, Electronic structures of doped $\mathrm{BaFe}_{2} \mathrm{As}_{2}$ materials: virtual crystal approximation versus super-cell approach, arXiv:1509.02635 (2015).

[25] I. M. Lifshitz, Anomalies of Electron Characteristics of a Metal in the High Pressure Region, Sov. Phys. JETP 11 (1960) 1130.

[26] S. Sharma et al., Structural investigations in $\mathrm{BaFe}_{2 x} \mathrm{Ru}_{x} \mathrm{As}_{2}$ as a function of $\mathrm{Ru}$ and temperature, Acta. Cryst. B 71 (2015) 61.

[27] C. Liu et al., Importance of the Fermi-surface topology to the superconducting state of the electron-doped pnictide $\mathrm{Ba}\left(\mathrm{Fe}_{1-x} \mathrm{Co}_{x}\right)_{2} \mathrm{As}_{2}$, Phys. Rev. B 84 (2011) 020509(R).

[28] S. N. Khan et al., Lifshitz Transition and Chemical Instabilities in $\mathrm{Ba}_{1-x} \mathrm{~K}_{x} \mathrm{Fe}_{2} \mathrm{As}_{2}$ Superconductors, Phys. Rev. Lett. 112 (2014) 156401.

[29] J. Fink et al., Electronic structure studies of $\mathrm{BaFe}_{2} \mathrm{As}_{2}$ by angle-resolved photoemission spectroscopy, Phys. Rev. B 79 (2009) 155118.

[30] N. Ni et al., Anisotropic thermodynamic and transport properties of single-crystalline $\mathrm{Ba}_{1-x} \mathrm{~K}_{x} \mathrm{Fe}_{2} \mathrm{As}_{2}$ ( $\mathrm{x}=0$ and 0.45), Phys. Rev. B 78 (2008) 014507.

[31] Y. Su et al., Antiferromagnetic ordering and structural phase transition in $\mathrm{BaFe}_{2} \mathrm{As}_{2}$ with $\mathrm{Sn}$ incorporated from the growth flux, Phys. Rev. Lett. 79 (2009) 064504.

[32] S.-Y. Xu et al., Lifshitz transition and Van Hove singularity in a three-dimensional topological Dirac semimetal, Phys. Rev. B 92 (2015) 075115 .

[33] Y. Lemonik et al., Spontaneous symmetry breaking and Lifshitz transition in bilayer graphene, Phys. Rev. B 82 (2010) 201408(R). 
[34] A. Varlet et al., Anomalous Sequence of Quantum Hall Liquids Revealing a Tunable Lifshitz Transition in Bilayer Graphene, Phys. Rev. Lett. 113 (2014) 116602.

[35] D. LeBoeuf et al., Lifshitz critical point in the cuprate superconductor $\mathrm{YBa}_{2} \mathrm{Cu}_{3} \mathrm{O}_{y}$ from high-field Hall effect measurements, Phys. Rev. B 83 (2011) 054506.

[36] N. Xu, P. Richard, X. Shi, A. van Roekeghem, T. Qian, E. Razzoli, E. Rienks, G.-F. Chen, E. Ieki, K. Nakayama, T. Sato, T. Takahashi, M. Shi, and H. Ding, Possible nodal superconducting gap and Lifshitz transition in heavily hole-doped $\mathrm{Ba}_{0.1} \mathrm{~K}_{0.9} \mathrm{Fe}_{2} \mathrm{As}_{2}$, Phys. Rev. B 88, (2013) 220508(R); Y. Liu, and T. A. Lograsso, Crossover in the magnetic response of single-crystalline $\mathrm{Ba}_{1-x} \mathrm{~K}_{x} \mathrm{Fe}_{2} \mathrm{As}_{2}$ and Lifshitz critical point evidenced by Hall effect, Phys. Rev. B 90, (2014) 224508.

[37] S. Ideta, T. Yoshida, I. Nishi, A. Fujimori, Y. Kotani, K. Ono, Y. Nakashima, S. Yamaichi, T. Sasagawa, M. Nakajima, K. Kihou, Y. Tomioka, C. H. Lee, A. Iyo, H. Eisaki, T. Ito, S. Uchida, and R. Arita, Dependence of Carrier Doping on the Impurity Potential in TransitionMetal-Substituted FeAs-Based Superconductors, Phys. Rev. Lett. 110, (2013) 107007.

[38] C. Enderlein, S. M. Ramos, M. Bittencourt, M. A. Continentino, W. Brewer, and E. Baggio-Saitovich, Anomaly close to an electronic topological semimetal-insulator transition in elemental fcc-Yb under pressure, J. Appl. Phys 114,(2013) 143711.

[39] M. Rotter et al., Spin-density-wave anomaly at $140 \mathrm{~K}$ in the ternary iron arsenide $\mathrm{BaFe}_{2} \mathrm{As}_{2}$, Phys. Rev. B 78 (2008) 020503(R).

[40] M. Rotter, High-temperature superconductivity in doped $\mathrm{BaFe}_{2} \mathrm{As}_{2}$, PhD thesis, Ludwig-Maximilians-Universität Munchen (2010) 61.

[41] Smritijit Sen and Haranath Ghosh, Fermiology of 122 family of Fe-based superconductors: An ab initio study, Phys. Lett. A 379 (2015) 843.

[42] X. F. Wang et al., Anisotropy in the Electrical Resistivity and Susceptibility of Superconducting $\mathrm{BaFe}_{2} \mathrm{As}_{2}$ Single Crystals, Phys. Rev. Lett. 102 (2009) 117005. 
[43] E. Colombier et al., Electrical transport measurements under pressure for $\mathrm{BaFe}_{2} \mathrm{As}_{2}$ compounds doped with Co, Cr, or Sn, Supercond. Sci. Technol. 23 (2010) 054003.

[44] J. S. Kim et al., Specific heat anomalies for $\mathrm{T} \ll \mathrm{T}_{c}$ in superconducting single crystal doped $\mathrm{BaFe}_{2} \mathrm{As}_{2}$ : comparison of different flux growth methods, J. Phys.: Condens. Matter 21 (2009) 252201.

[45] S. J. Clark et al., First principles methods using CASTEP, Zeitschrift fuer Kristallographie 200 (2005) 567.

[46] J. P. Perdew et al., Generalized Gradient Approximation Made Simple, Phys. Rev. Lett. 77 (1996) 3865.

[47] M. Tegel et al., Structural and magnetic phase transitions in the ternary iron arsenides $\mathrm{SrFe}_{2} \mathrm{As}_{2}$ and $\mathrm{EuFe}_{2} \mathrm{As}_{2}$, J. Phys.: Condens. Matter 20 (2008) 452201.

[48] A. I. Goldman et al., Lattice and magnetic instabilities in $\mathrm{CaFe}_{2} \mathrm{As}_{2}$ : A single-crystal neutron diffraction study, Phys. Rev. B 78 (2008) 100506(R).

[49] L. Bellaiche and D. Vanderbilt, Virtual crystal approximation revisited: Application to dielectric and piezoelectric properties of perovskites, Phys. Rev. B 61 (2000) 7877.

[50] http://www.tcm.phy.cam.ac.uk/castep/documentation/WebHelp/content/modules/castep/th

[51] G. L. Sun et al., Single Crystal Growth and Effect of Doping on Structural, Transport and Magnetic Properties of $\mathrm{A}_{1-x} \mathrm{~K}_{x} \mathrm{Fe}_{2} \mathrm{As}_{2}$ (A = Ba, Sr), J. Supercond. Novel. Magn. 24(5) (2011) 1773. 